\title{
Serum levels of inflammasome pathway factors in clinically isolated syndrome and multiple sclerosis patients: a pilot study
}

\author{
YUSUF TAMAM ${ }^{1}$, HANDE YÜCEER ${ }^{2}$ BERNA DÜZEL ${ }^{1}$, MELIS ŞEN ${ }^{2}$ CANAN ULUSOY', \\ ERDEM TUZUN ${ }^{2}$
}

${ }^{1}$ Department of Neurology, Faculty of Medicine, Dicle University, Diyarbakır, Turkey

${ }^{2}$ Aziz Sancar Institute of Experimental Medicine, Istanbul University, Istanbul, Turkey

\begin{abstract}
Pathogenic roles of nuclear factor $\kappa B(N F-\kappa B)$ pathway and NLRP3 inflammasome complex factors are involved in multiple sclerosis (MS) development. Activation of the $N F-\kappa B, N L R P 3$, and caspase-1 cascade results in production of proinflammatory cytokines that lead to stimulation of macrophages, lymphocytes, and glial cells. Although increased levels of inflammasome complex factors are observed in MS, contribution of inflammasome pathway to conversion from clinically isolated syndrome (CIS) to relapsing remitting MS (RRMS) has been scarcely investigated. To examine predictive value of inflammasome factors in CIS-MS conversion, levels of NLRP3, caspase-1, and NF $\mathrm{K}$ are measured by ELISA in sera of age-gender matched CIS ( $n=18 ; 8$ converting, 10 non-converting) and RRMS $(n=23)$ patients. CIS and RRMS patients have comparable serum levels of NLRP3, caspase-1, and $N F \kappa B$. Similarly, no statistically significant difference can be found among converting and nonconverting CIS patients by means of inflammasome complex factor levels. Inflammasome factors are presumably overexpressed at early stages of MS. Therefore, they are unlikely to be used as biomarkers to predict CIS-MS conversion.
\end{abstract} $N F \kappa B$.

Key words: multiple sclerosis, clinically isolated syndrome, neuroinflammation, NLRP3, caspase-1,

(Cent Eur J Immunol 2020; 45 (2):237-240)

\section{Introduction}

Blood-brain barrier (BBB) disruption and microglial activation are general pathological features of various neurologic diseases including multiple sclerosis (MS). When immune tolerance gets impaired in MS patients, myelin specific T cells become activated, break through the BBB, gain access into the brain, and increase in number to initiate an autoimmune response against myelin proteins [1]. The nuclear factor NF- $\mathrm{B}$ pathway enhances the expression of proinflammatory genes including nucleotide-binding oligomerization domain/leucine-rich repeat, and pyrin domain containing 3 (NLRP3) multiprotein complex [1-3]. NLRP3 inflammasome structurally transforms into an active inflammasome complex by integrating caspase- 1 enzyme. Activated caspase- 1 triggers the release of pro-inflammatory cytokines IL-1b and IL-18. These pro-inflammatory cytokines lead to the stimulation of microglia, macrophages, astrocytes, and recruitment of
B cells [4]. Pathogenic role of NLRP3 inflammasome has been shown in MS development. NFkB and NLRP3 are highly activated at sites of inflammation in MS. Also, an increased expression levels of caspase-1, IL-1b, and IL-18 are detected in glial scar or sclerosis $[5,6]$.

In this study, we aimed to compare serum levels of inflammasome complex-associated inflammatory mediators (NLRP3, caspase-1, and NF- $\kappa \mathrm{B}$ ) in relapsing-remitting MS (RRMS) and clinically isolated syndrome (CIS) patients to find out whether these mediators can be used as biomarkers to predict CIS-MS transition.

\section{Material and methods}

\section{Participants}

The study included 23 RRMS (32.9 \pm 6.8 -year-old; 14 women, 9 men) and 18 age/gender-matched CIS (30.8 \pm 6.9 -year-old; 11 women, 7 men) patients (Table 1 ). $\mathrm{Pa}$ -

Correspondence: Yusuf Tamam MD, Department of Neurology, Faculty of Medicine, Dicle University, 21280 Kitilbil, 21280 Diyarbakir,

Turkey, e-mail: drerdem@yahoo.com

Submitted: 28.12.2017; Accepted: 18.04.2018 
Table 1. Demographic and clinical characteristics of relapsing-remitting multiple sclerosis (RRMS) and clinical isolated syndrome (CIS) patients during blood sampling

\begin{tabular}{|c|c|c|c|c|}
\hline Characteristic & $\begin{array}{l}\text { RRMS } \\
(n=23)\end{array}$ & $\begin{array}{c}\text { Converting CIS } \\
\quad(n=8)\end{array}$ & $\begin{array}{c}\text { Non-converting CIS } \\
(n=10)\end{array}$ & $p$-value \\
\hline Age & $32.9 \pm 6.8$ & $29.5 \pm 6.9$ & $31.9 \pm 6.9$ & $0.417^{*}$ \\
\hline Gender (women/men) & $14 / 9$ & $5 / 3$ & $6 / 4$ & $0.994^{* *}$ \\
\hline Disease duration (years) & $6.8 \pm 3.2$ & - & - & - \\
\hline Number of relapses & $5.2 \pm 1.4$ & $1.0 \pm 0.0$ & $1.0 \pm 0.0$ & $<0.001^{* *}$ \\
\hline Annualized relapse rate & $1.0 \pm 0.7$ & - & - & - \\
\hline Number of MRI lesions & $6.1 \pm 2.1$ & $1.8 \pm 0.7$ & $1.9 \pm 0.8$ & $<0.001^{* *}$ \\
\hline EDSS score & $3.7 \pm 0.6$ & $1.7 \pm 0.4$ & $1.8 \pm 0.3$ & $<0.001^{* * *}$ \\
\hline Progression index & $0.7 \pm 0.3$ & - & - & - \\
\hline
\end{tabular}

tients' diagnosis was based on McDonald's criteria [7]. CIS patients were followed up by the same neurologist for five years, with planned examinations every 6 months and whenever a relapse was suspected. They were divided into two groups as CIS patients that converted to MS any time within the 5-year follow-up period as per McDonald criteria $(n=8)$, and as CIS patients that did not convert to MS $(n=10)$ within the same timeframe. All patients were under remission during blood sample collection (i.e. sera of CIS patients were collected 2-3 months after their initial attack, while they were in remission period). No immunosuppressive treatment (e.g. corticosteroids, azathioprine) was given to the patients for at least two months prior to blood sample collection. None of the CIS patients were under immunomodulating drug treatment, whereas all MS patients were using immunomodulating medications (12 interferon $\beta, 7$ glatiramer acetate, and 4 fingolimod). All sera were collected between 8:00-10:00 a.m. and stored at $-80^{\circ} \mathrm{C}$. The study was approved by the institutional review board and all patients gave written informed consent.

\section{ELISA}

Serum levels of NLRP3, NFKB, and caspase-1 were determined by ELISA kits (MyBioSource, San Diego, CA, USA) as per manufacturer's recommendations. The results were obtained as OD values and were converted to $\mathrm{ng} / \mathrm{ml}$ or $\mathrm{pg} / \mathrm{ml}$ under the guidance of curves generated from the values of standards.

\section{Statistical analysis}

ANOVA or Student's $t$-test were used for comparison of quantitative variables, whereas non-quantitative variables were compared with Kruskal-Wallis test. $\chi^{2}$ test was used for comparison of gender prevalence. Correlation statistics were performed with Pearson and Spearman correlation tests, as required. Statistically significant differences were considered as $p<0.05$.

\section{Results}

\section{Patient characteristics}

RRMS, converting CIS, and non-converting CIS patients displayed comparable age and gender distributions. EDSS ( $3.7 \pm 0.6$ vs. $1.7 \pm 0.4$ and $1.8 \pm 0.3, p<0.001)$, relapse numbers $(5.2 \pm 1.4$ vs. $1.0 \pm 0.0$ and $1.0 \pm 0.0$, $p<0.001)$, and MRI lesion numbers $(6.1 \pm 2.1$ vs. $1.8 \pm 0.7$ and $1.9 \pm 0.8, p<0.001)$ of RRMS patients were higher than converting and non-converting CIS patients, as expected. Average annualized relapse rate and progression index values of MS patients were $1.0 \pm 0.7$ and $0.7 \pm 0.3$, respectively (Table 1).

\section{Serum levels of inflammatory mediators}

There were no significant differences among CIS and RRMS patients by means of serum levels of NLRP3 $(p=0.373), \mathrm{NF \kappa B}(p=0.141)$, and caspase- $1(p=$ 0.249) (Fig. 1, upper panels). Likewise, converting and non-converting CIS patients showed comparable NLRP3 $(p=0.261), \mathrm{NF}-\kappa \mathrm{B}(p=0.751)$, and caspase-1 $(p=0.776)$ levels (Fig. 1, lower panels). Although levels of inflammatory mediators were also compared among MS patients receiving different immunomodulating medications, no difference could be found among patients under interferon $\beta$, glatiramer acetate, and fingolimod treatment (data not shown). No significant correlation was observed between serum NLRP3, NF- $\kappa$ B, and caspase-1 levels and age, disease duration, relapse number, MRI lesion number, and EDSS variables.

\section{Discussion}

The impact of inflammatory mediators like NLRP3, caspase-1, and NFKB in MS development and pathogenesis is well-known [4-6]. However, the link between these mediators and CIS has never been studied. In this study, serum levels 

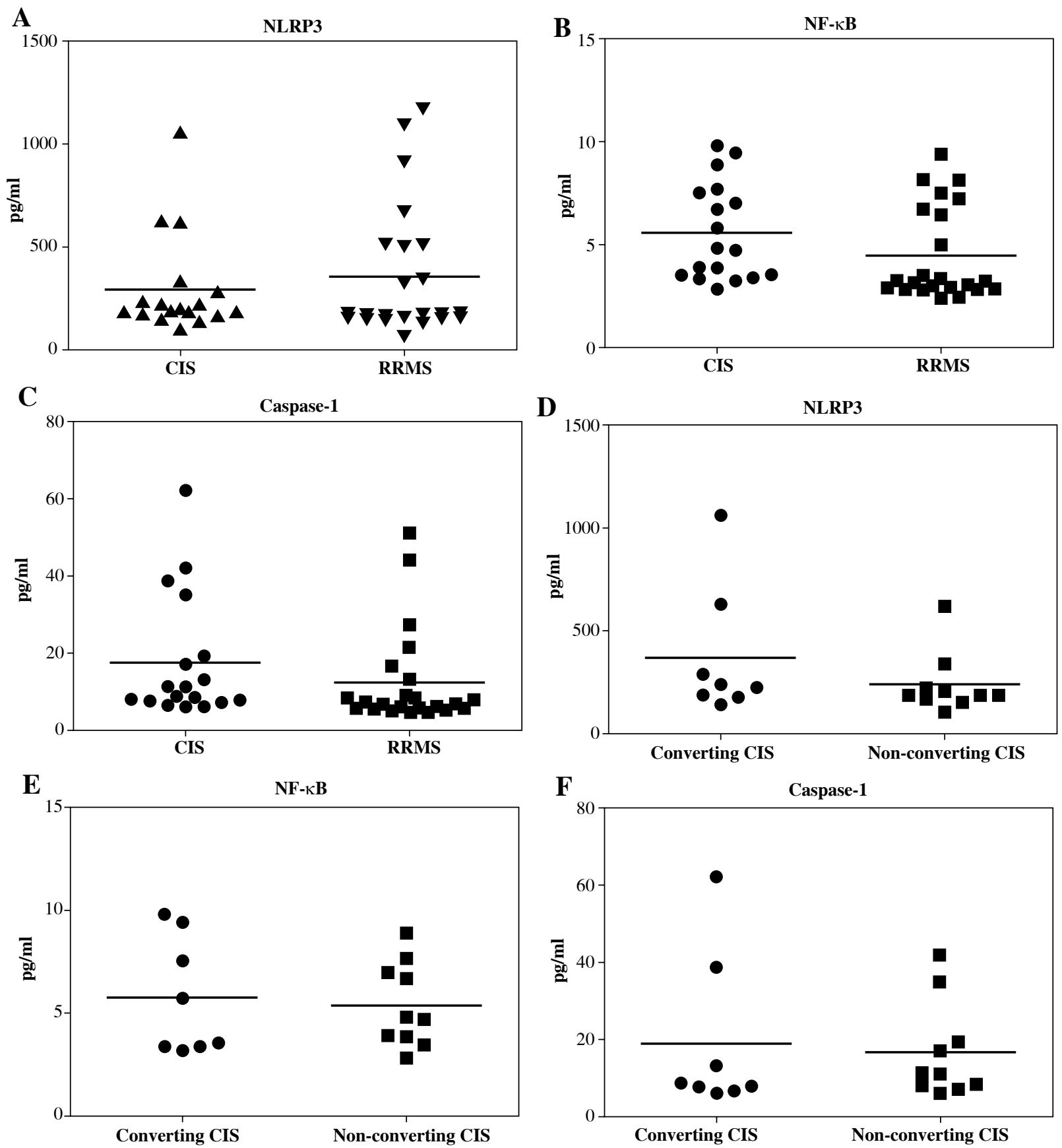

Fig. 1. Comparison of serum NLRP3, NF- $\kappa B$, and caspase-1 levels of patients with clinically isolated syndrome (CIS) vs. relapsing-remitting multiple sclerosis (RRMS) (upper panels) and converting vs. non-converting CIS (lower panels). Horizontal lines indicate mean values. No significant difference could be found between study groups by Student's $t$-test

of NLRP3, caspase-1, and NFKB in RRMS patients were not found significantly different from CIS, despite well-known involvement of microglial activation in MS. Therefore, serum levels of inflammasome complex factors are unlikely to be used as biomarkers to predict conversion from CIS to MS. In compliance with our results, a previous study has failed to find a difference between peripheral blood cell mRNA expression levels of inflammasome complex factors in converting and non-converting CIS patients [8]. Our findings also suggest that basic immunomodulatory treatments do not affect levels of NLRP3, NFKB, and caspase-1. However, a few cases were rather low in each treatment group in our pilot study, and thus future studies with larger cohorts are required for a decisive conclusion on this issue. 
A potential reason for lack of difference between CIS and RRMS groups could be the enhancement of neuroinflammation pathway factors in very early stages of the disease. There are various controversial hypotheses stating that inflammatory factors involved in neurodegeneration can also be engaged in neuronal regeneration and remyelination [9]. For instance, activated astrocytes cause apoptosis of infiltrating $\mathrm{T}$ cells by releasing IL-27, the trophic factor modulating neuronal regeneration. Furthermore, M2 phenotype microglia take a major part in neuroprotection and remyelination of axons [10]. Similarly, activated $\mathrm{NF}-\kappa \mathrm{B}$ in neurons also has a neuroprotective effect through induction of anti-apoptotic genes and proteins regulating synaptic plasticity [9]. Therefore, the association between inflammation pathways and MS progression might be more complex than anticipated, and studies measuring levels of inflammatory mediators might be limited by the complex nature of MS.

The authors declare no conflict of interest.

\section{References}

1. Zhang X, Tao Y, Chopra M, et. al. (2015): IL-11 induces Th17 cell responses in patients with early relapsing-remitting multiple sclerosis. J Immunol 194: 5139-5149.

2. Leibowitz SM, Yan J (2016): NF-кB pathways in the pathogenesis of multiple sclerosis and the therapeutic implications. Front Mol Neurosci 9: 84.

3. Mattson MP, Camandola S (2001): NF-kappaB in neuronal plasticity and neurodegenerative disorders. J Clin Invest 107 : 247-254.

4. Song L, Pei L, Yao S, et. al. (2017): NLRP3 inflammasome in neurological diseases, from functions to therapies. Front Cell Neurosci 11: 63.

5. Inoue M, Shinohara ML (2013): NLRP3 Inflammasome and MS/EAE. Autoimmune Dis 2013: 859145.

6. Mc Guire C, Prinz M, Beyaert R, van Loo G (2013): Nuclear factor kappa B (NF- $\kappa \mathrm{B})$ in multiple sclerosis pathology. Trends Mol Med 19: 604-613.

7. Polman CH, Reingold SC, Banwell B (2011): Diagnostic criteria for multiple sclerosis: 2010 revisions to the McDonald criteria. Ann Neurol 69: 292-302.

8. Hagman S, Kolasa M, Basnyat P (2015): Analysis of apoptosis-related genes in patients with clinically isolated syndrome and their association with conversion to multiple sclerosis. J Neuroimmunol 280: 43-48.

9. Srinivasan M, Lahiri DK (2015): Significance of NF-кB as a pivotal therapeutic target in the neurodegenerative pathologies of Alzheimer's disease and multiple sclerosis. Expert Opin Ther Targets 19: 471-487.

10. Thompson KK, Tsirka SE (2017): The diverse roles of microglia in the neurodegenerative aspects of central nervous system (CNS) autoimmunity. Int J Mol Sci 18: pii: E504. 